PROCEEDINGS OF THE

AMERICAN MATHEMATICAL SOCIETY

Volume 126, Number 8, August 1998, Pages 2477-2479

S 0002-9939(98)04448-7

\title{
SOME STRUCTURE OF BOREL LOCALES
}

\author{
JOHN ISBELL \\ (Communicated by Alan Dow)
}

\begin{abstract}
All Borel classes of sublocales of the real line after the first ambiguous class (in particular, the limit ambiguous classes) have proper (=irreducible) representatives.
\end{abstract}

\section{INTRODUCTION}

This paper is very closely connected to my previous paper [2] and to Till Plewe's paper [4]. It is probably not advisable to try to read this paper without reading at least the introduction of [2]. Nothing here depends on Plewe's work; but much of the interest of this paper does depend on [4].

Of course, "Borel locale", like "Borel set", is a relative concept; but "Borel set" is often used in an absolute sense, meaning a space homeomorphic with a Borel set in some complete metric space. Here "Borel locale" is used in the same way. But most Borel sets are not Borel locales. The Borel sublocales of, say, the real line $\mathbf{R}$, are defined like Borel subsets, generated from open or closed sets by countable join and countable meet. The joins and meets are taken in the lattice of sublocales. It turns out - this is a major result of Plewe's [4] - that an $F_{\sigma \delta}$ Borel set which is not $G_{\delta \sigma}$, in $\mathbf{R}$, is never an $F_{\sigma \delta}$ sublocale.

In Borel locale theory we use the " $F_{\sigma}, F_{\sigma \delta}, \ldots$ " notation, but we write $O_{\delta}, O_{\delta \sigma}, \ldots$ for $G_{\delta}, G_{\delta \sigma}, \ldots$ Also Kuratowski's terminology, of additive, multiplicative, and ambiguous Borel class $\alpha$ for each ordinal $\alpha<\omega_{1}$, is used. Now the culmination of the previous paper [2] is a list of four problems, on one of which (Problem 4) there has been no progress.

Problem 1. Does the real line $\mathbf{R}$ have proper (=irreducible) representatives $S$ of each Borel class $\mathcal{B}$ of sublocales? That is, $S \in \mathcal{B}, S \neq \varnothing$, and no nonzero open sublocale of $S$ belongs to a lower Borel class.

This was answered in [2] except for ambiguous class $\lambda$ where $\lambda$ is a limit ordinal. This paper will remove the exception. The answer is negative for the first ambiguous class - an $F_{\sigma}$ and $G_{\delta}$ has an open subset open in $\mathbf{R}$ or an open subset closed in $\mathbf{R}$-but otherwise affirmative.

Problem 2. If a metrizable locale is Borel in every metrizable extension, must it be spatial?

Received by the editors January 6, 1997

1991 Mathematics Subject Classification. Primary 54A05, 54H05; Secondary 04A15.

Key words and phrases. Irreducible Borel sublocale.

(C)1998 American Mathematical Society 
Being spatial is sufficient; that is, a metric space which is a Borel sublocale (not just a Borel set) in some completion is Borel in every metric extension [2]. Plewe has solved Problem 2 affirmatively, and more: a locale which is Borel in every completion is spatial $[4,7.8]$.

Problem 3. Is every spatial Borel sublocale of $\mathbf{R}$ an $O_{\delta \sigma}$ ?

Plewe has about "half" of this problem. As stated above, a spatial $F_{\sigma \delta}$ must be $O_{\delta \sigma}$. Indeed a spatial $O_{\delta \sigma \delta}$ must be $O_{\delta \sigma}[4,7.12]$.

\section{AMBiguous LIMIT ClASSES}

All locales considered will be (unless an exception is clearly stated) metrizable. ("Locales" include spaces.) The terminology for Borel sublocales [2] is basically after Kuratowski [3] for Borel sets; but we say ' $O_{\delta}$ ' instead of ' $G_{\delta}$ ', because a countable meet of open subspaces of a metrizable space need not be a subspace. A join of subspaces is a subspace, so we stay with ' $F_{\sigma}$ '. Of course $F_{\sigma \delta}$ 's, $O_{\delta \sigma}$ 's and so on need not be subspaces; even in $\mathbf{R}$, where (as in any complete space) $O_{\delta}=G_{\delta}, F_{\sigma \delta}$ 's are usually not subspaces. It is not known whether every Borel subspace of $\mathbf{R}$ is an $O_{\delta \sigma}$.

If these novelties unsettle the reader, put down this paper. If still interested in Borel sublocales, go to [1] - where a theorem of Hausdorff and Montgomery is extended from complete spaces to arbitrary spaces (and metrizable locales) by replacing ' $G_{\delta}$ ' with ' $O_{\delta}$ '.

A Borel sublocale $B$ of a locale $A$ is said to be properly of Borel class $\mathcal{B}$, higher than ambiguous class $1\left(F_{\sigma}\right.$ and $\left.O_{\delta}\right)$, if $B$ is of class $\mathcal{B}, B \neq 0$, and $B$ is nowhere locally Borel of any lower class than $\mathcal{B}$. Ambiguous class 1 is excluded because it is impossible, at least in metrizable spaces; a nonzero $F_{\sigma}$ and $O_{\delta}$ sublocale must be locally closed at some of its points [2, p. 259].

"Properly of class $\mathcal{B}$ " in the Borel-set (Russian) literature is "irreducible of class $\mathcal{B}$ ". It seems to me that here a systematic difference between Borel-set and Borellocale terminology is a positive advantage. (There is also a clash between "irreducible" and Hausdorff's reducible sets - which we call, following [3], developable.)

In [2] it was shown that the Cantor set $\mathbf{C}$, and thus anything containing $\mathbf{C}$, has sublocales properly of Borel class $\mathcal{B}$ for every class $\mathcal{B}$ higher than ambiguous class 1 except perhaps the limit ambiguous classes. The exception will now be removed.

Let $\alpha$ be any nonzero countable limit ordinal. Let $\left(\beta_{n}\right)$ be an increasing sequence of ordinals greater than zero with limit $\alpha$. By [2], $\mathbf{C}$ has sublocales $B_{n}$ properly of multiplicative Borel class $\beta_{n}$. Since $\beta_{n}>0, B_{n}$ has no isolated point; therefore its closure $B_{n}^{-}$in $\mathbf{C}$ is homeomorphic with $\mathbf{C}$. Now observe that $\mathbf{C}$ is homeomorphic with $\mathbf{C} \times \mathbf{C}$. In $\mathbf{C} \times \mathbf{C}$ we describe a sublocale $B$, contained in $Q \times \mathbf{C}$ where $Q=\left\{p_{n}\right.$ : $n<\omega\}$ is some countable dense subset of $\mathbf{C}$. For each $n$, use some homeomorphism $h: B_{n}^{-} \rightarrow\left\{p_{n}\right\} \times \mathbf{C}$ to identify $B_{n}$ with a dense sublocale $D_{n}$ of $\left\{p_{n}\right\} \times \mathbf{C}$; since $h$ maps a closed set homeomorphically, $D_{n}$ is properly of multiplicative Borel class $\beta_{n}$ in $\mathbf{C} \times \mathbf{C}$. Let $E$ be $p l(Q) \times \mathbf{C}$. Let $B$ be the join of $E$ and all $D_{n}$. Since each $D_{n}$ is Borel of additive class $\alpha$ (indeed, of a lower class), and $E$ is $F_{\sigma \delta}, B$ is Borel of additive class $\alpha$. But $B$ is also, we shall show, the meet of Borel sublocales $S_{n}$ described as follows. $T_{n}$ is the $F_{\sigma} Q_{n} \times \mathbf{C}$ where $Q_{n}=\left\{p_{m}: m>n\right\} . S_{n}$ is $T_{n} \vee D_{1} \vee D_{2} \vee \cdots \vee D_{n}$. So $S_{n}$ is Borel of multiplicative class $\alpha$.

Each $S_{n} \geq B$ since $T_{n} \geq E$ and each $D_{m}$ is either a summand of $S_{n}(m \leq n)$ or $\leq T_{n} ;$ so $M=\bigwedge S_{n} \geq B$. To show that $M=B$, we need only show that for each 
nonzero closed subspace $H$ of $Q \times \mathbf{C}$ disjoint from $B$, not all $S_{n} \wedge H$ are dense in $H[1,1.5]$. Now since $\mathbf{C}$ is compact, the projection $I$ of $H$ in $Q$ is closed. But $H$ is disjoint from $B$ and thus from $E=p l(Q) \times \mathbf{C}$, so $I \wedge p l(Q)=0$. Thus $I$ has no nonzero pointless part; $I$ is scattered, and in particular it has an isolated point $p_{n}$. Then $H \wedge\left(\left\{p_{n}\right\} \times \mathbf{C}\right)$ is a nonzero open subspace $U$ of $H$. $H$ is disjoint from $D_{n}$, so $U$ is too, and as $U \subset\left\{p_{n}\right\} \times \mathbf{C}$ this makes $U$ disjoint from $S_{n}$. Since $U$ is nonzero open in $H$, this means $S_{n} \wedge H$ is not dense.

Now $M=B$ is of ambiguous class $\alpha$. Every nonzero open part $V$ of it has nonzero meet with $\left\{p_{n}\right\} \times \mathbf{C}$ for arbitrarily large $n$ (since $D_{n}$ is dense in $\left\{p_{n}\right\} \times \mathbf{C}$ ); that is, closed in $V$ and of no Borel class lower than multiplicative class $\beta_{n}$, so $B$ is properly of ambiguous class $\alpha$.

Proposition. For every Borel class $\mathcal{B}$ higher than ambiguous class $1, \mathbf{C}$ has a sublocale properly of class $\mathcal{B}$.

\section{REFERENCES}

[1] J. Isbell, First steps in descriptive theory of locales, Trans. Amer. Math. Soc. 327 (1991), 353-371; Corrections, 341 (1994), 467-468. MR 92b:54078; MR 94c:54071

$[2]$, Some problems in descriptive locale theory, Canadian Math. Soc. Conference Proceeding 13 (1992), 243-265. MR 94a:54091

[3] K. Kuratowski, Topologie, vol. I, 4th ed., Warsaw, 1958. MR 19:873d

[4] T. Plewe, Localic products of spaces, Proc. London Math. Soc. (3) 73 (1996), 642-678. MR 97d:54012

Department of Mathematics, State University of New York at Buffalo, Buffalo, NEW York 14214

E-mail address: ji2@acsu.buffalo.edu 